\title{
A RATIONAL APPROACH FOR MODELLING THE MECHANI- CAL BEHAVIOUR OF MATERIALS EXHIBITING SYMMETRY RELATIONS
}

\author{
VINCENT MAGNENET, RACHID RAHOUADJ AND JEAN-FRANÇOIS \\ GANGHOFFER
}

Presented by Jean-François Ganghoffer

\begin{abstract}
A novel and rational approach based on Lie analysis is proposed to investigate the mechanical behaviour of materials exhibiting experimental master curves. This approach provides a priori two ways of formulating constitutive laws from data as well as the possibility of predicting new master curves and material charts. The first part of the paper is devoted to the presentation of the algorithm. Afterwards, the strategy is applied to the uniaxial creep and rupture behaviour of a Chrome-Molybdene alloy $(9 \mathrm{Cr} 1 \mathrm{Mo})$ at different temperatures and stress levels.
\end{abstract}

\section{Contents}

1 Introduction $\quad \mathbf{3 0}$

2 General Strategy 35

2.1 Step 1: Formulation of Symmetries From Experimental Data . . . 35

2.2 Step 2: Enumeration and Classification of Symmetry Conditions . 38

2.3 Step 3: Formulation of the Constitutive Equations $\boldsymbol{\Delta}$. . . . . . . 39

2.4 Step 4: Computation of the Lie Algebras and Predictions . . . . . 40

3 Application to the 9Cr1Mo Creep: Observed Symmetries 42

3.1 Step $1 \ldots \ldots \ldots \ldots$. . . . . . . . . . . . . . . 42

3.1.1. Rupture Curves . . . . . . . . . . . . . . . . . 42

3.1.2. Creep Curves . . . . . . . . . . . . . . . 44

3.2 Step $2 \ldots \ldots \ldots \ldots \ldots$. . . . . . . . . . . . . . 46

3.3 Step $3 \ldots \ldots \ldots \ldots \ldots$. . . . . . . . . . . . . . 47

3.4 Step $4 \ldots \ldots \ldots \ldots \ldots \ldots$ 
3.4.1. Lie Algebra of the Creep Model . . . . . . . . . . . . 49

3.4.2. Lie Algebra of Equation (63) . . . . . . . . . . . 50

\section{Discussion and Concluding Remarks}

\section{Introduction}

Invariance relations - together with master curves as their graphical counterpart are currently used in rheology to synthesize the constitutive response of various materials submitted to mechanical or thermal solicitations. A typical example of such an empirical construction is the well-known time-temperature equivalence principle, stating an equivalence between the effect of time and of temperature, as proposed originally by Williams, Landel and Ferry, giving rise to the so-called WLF model. In the same spirit, power laws in fatigue have been formulated in [2,3] (see also [5] and references therein), using arguments of dimensional analysis in combination with similarity principles.

One important field in which invariant relations are used is high temperature creep of metallic alloys, devoted to industrial applications. Landmarks in this context are the Larson-Miller and the Dorn models (see e.g. [22] and the references therein). Those relations intrinsically have the meaning of invariants, and further allow to extrapolate experimental data at different temperatures, assuming that the microstructure of the material remains stable during the creep test. The two main objectives of this kind of investigation are either the determination of the time needed to reach a given strain for fixed stress and temperature, or the estimation of the time leading to rupture, in the same controlled conditions. In principle, both problems, although interrelated (viscoplasticity is coupled to damage), are distinct: the first one is a creep problem, whereas the second more specifically involves damage. Hence, the iso-strain responses in a double logarithmic representation of stress vs time are not able to include the limit case of rupture, since the rupture curves are not iso-strain curves in this representation. We consider in the present study the creep behaviour of the $9 \mathrm{Cr} 1 \mathrm{Mo}$ martensitic stainless steel, which is also known for its good thermal-fatigue strength and oxydation resistance [10]. These structural components of nuclear power plants are particularly operative at high temperature. According to specialists in the field, one of the most crucial problem in determining the integrity of structural components is the creep behaviour [11]. Indeed, due to thermal activation, the material can slowly and continuously deteriorate under constant stress, even for low stress levels, as mentioned by [27]. In [1], the behaviour of the $9 \mathrm{Cr} 1 \mathrm{Mo}$ alloy at the three temperatures $923 \mathrm{~K}, 873 \mathrm{~K}$ and $823 \mathrm{~K}$ is 
analyzed. The authors state that Norton law

$$
\dot{\varepsilon}=A \sigma^{n} \exp \left(-\frac{Q}{R T}\right)
$$

in which $A$ is a constant (see the nomenclature for the significance of other symbols) is well representative for this material, especially regarding the activation energy $Q$. According to the same authors, the Monkman-Grant relation (MGR), descriptive of a rupture criterion, well describes the relation between the minimum creep rate (secondary stage) and the rupture time. In parallel to this, they examine the microstructural evolution of the carbide precipitation during creep loading, and relate the creep resistance to the coarsening of certain precipitates. A log-log plotting of the rupture time, $t_{R}$, versus the steady-state creep rate (or the minimum creep rate $\dot{\varepsilon}_{\text {min }}$ ), shows empirically a straight line, hence one may write

$$
\log t_{R}+m \log \dot{\varepsilon}_{\min }=C
$$

with $m, C$ some constants. In practice, one very often considers the modified Monkman-Grant relation (abbreviated MMGR)

$$
\log \left(\frac{t_{R}}{\varepsilon_{R}}\right)+m^{\prime} \log \dot{\varepsilon}_{\min }=C^{\prime}
$$

in which the strain to fracture $\varepsilon_{R}$ is closely related to creep deformation processes that lead to the formation of cracks and cavities.

In fact, the MMGR has been introduced by [8] to account for tertiary creep, and to better describe experimental results for certain metals and alloys. In [19], an interpretation of the MGR and the MMGR is provided, following a simple mathematical analysis of the strain rate versus time responses. This analysis has been applied to the Zircaloy-4 alloy and the stainless steel AISI 304. The same author concludes that the MGR and the MMGR relations result from a too coarse approximation of the area sustained by the strain rate versus time curve, and from the adjustment of experimental data in a double logarithmic representation. However, those relations (MGR and MMGR) remain very useful when evaluating order of magnitudes of quantities such as $\varepsilon_{R}, t_{R}$ or $\dot{\varepsilon}_{\text {min }}$. The authors of [10] considered also the 9Cr1Mo alloy as well as the stainless austenitic steel 316LN, in a temperature range between $823 \mathrm{~K}$ and $923 \mathrm{~K}$. They analyze experimental results from the MGR and MMGR relations, concluding that the MMGR model is in quite good adequation with the measurements. Based on a continuum creep damage mechanics approach, a new relationship between time to reach MGR ductility and rupture life in terms of damage tolerance factor is proposed in [18]. The authors showed the validity of this relationship for creep data relative to 9Cr1Mo and AISI 304 
stainless steels, with implications to tertiary creep damage and engineering creep design.

In practice, many other empirical relations involving invariants can be encountered. For instance, if we start from the expression of the thermally activated creep strain rate

$$
\dot{\varepsilon}=\dot{\varepsilon}_{0} \exp \left(-\frac{Q}{R T}\right)
$$

and further identify the global material response to the secondary creep regime with $\dot{\varepsilon}_{0}$ the constant minimum creep rate (the primary and tertiary creep stages are neglected), we obtain

$$
t=\frac{C}{\dot{\varepsilon}_{0}} \exp \left(\frac{Q}{R T}\right)
$$

This relation can be rearranged into the form

$$
\log t=\frac{B_{1}}{T}-\log B_{2}, \quad \text { with } B_{1}=\frac{Q}{R}, \quad \text { and } \quad B_{2}=\frac{\dot{\varepsilon}_{0}}{C} .
$$

As underlined in [22], the Larson-Miller parameter $P_{L M}$ results from the assumption that solely $Q$ is a function of stress, while $B_{2}$ is constant, hence the relationship

$$
P_{L M}=T\left(\log t+\log B_{2}\right)
$$

with the parameter $P_{L M}$ a function of the applied stress. Opposite to this model, the Dorn parameter $P_{D}$ is obtained when the activation energy is assumed to be stress independent, while $B_{2}$ is a function of stress, thus giving

$$
P_{D}=\frac{B_{1}}{T}-\log t
$$

with the parameter $P_{D}$ also a function of the applied stress. Both Larson-Miller and Dorn parameters are of great interest to extrapolate experimental data if the value of some loading parameter (e.g. temperature, applied stress) cannot be reached in an experimental context. These kind of relationships, including the MGR and MMGR relations written in the same spirit - see Table 1 for a brief literature review - were at their time formulated in a purely empirical manner so as to fit experimental responses.

They are closely related to the notion of master curve, which is nothing but the graphical consequence of the invariance property in a given representation (choice of a set of variables to define a planar representation). Despite the great practical interest of these relationships, the mathematical background providing the suitable tools to predict invariance relations and the associated master curves has not yet been fully developed to our knowledge. 
Table 1. Some creep invariants encountered in the literature.

\begin{tabular}{ll} 
Invariant(s) & Authors \\
\hline$T\left(\log t+\log B_{2}\right)$ & Larson \& Miller \\
$\frac{B_{1}}{T}-\log t$ & Dorn \\
$\log t_{R}+m \log \dot{\varepsilon}_{\text {min }}$ & Monkman-Grant \\
$\log \left(\frac{t_{R}}{\varepsilon_{R}}\right)+m^{\prime} \log \dot{\varepsilon}_{\text {min }}$ & Modified Monkman-Grant
\end{tabular}

This lack of theoretical framework may be remedied by considering the Lie symmetry analysis as a relevant mathematical tool to analyze and predict master curves and their associated invariants. By "master curve", we mean a graphical superposition of different experimental curves in accordance with some geometrical mapping. For instance, the so-called time-temperature equivalence principle reveals the possibility of plotting a unique master curve from several isothermal mechanical responses of a polymeric-like material. More precisely, if we consider different plots representing the logarithm of the isothermal creep compliance $J(t, T)$ - defined as the ratio of the Henky strain $\varepsilon(t)$ to the Cauchy stress $\sigma(t)$ at a given time $t$ and temperature $T$ - vs the logarithm of time $\log t$, a unique curve $C$ can be obtained by shifting the different isothermal curves along the $\log t$ axis. The resulting curve $C$ is called the master curve, but the expression can also refer to the geometrical mapping allowing the construction of $C$ (a horizontal translation in the present case). Beyond the geometrical nature of the mapping, the master curve is by essence governed by three principles closely related to the three basic axioms of the Lie groups theory. Indeed, if we denote by $C_{T}$ the isothermal plot of $\log J(t, T)$ vs $\log t$ at a given temperature $T$, we have

- any creep compliance curve $C_{T}$ is obtained by translating itself by 0 along the $\log t$ axis. This particular translation is nothing but the identity application, which is in fact the neutral element of translations (in the sense of composition).

- if $C_{T^{\prime}}$ is obtained from $C_{T}$ by a translation of $\mu$ along the $\log t$ axis, then $C_{T}$ can be obtained by shifting $C_{T^{\prime}}$ of $-\mu$ along the same axis. This property can be viewed as a reflexivity property.

- if $C_{T^{\prime \prime}}$ is obtained from $C_{T^{\prime}}$ by a translation of $\mu^{\prime}$ along the $\log t$ axis, and if $C_{T^{\prime}}$ is obtained from $C_{T}$ by a translation of $\mu$, then $C_{T^{\prime \prime}}$ can be obviously obtained by translating $C_{T}$ of $\mu^{\prime}+\mu$ (transitivity property). 
In this example, the scalar $\mu$ represents the Lie group parameter, allowing a continuous mapping from one curve to another one which corresponds to the length of the translation vector. In a more general case, the geometrical mapping leading to a master curve may be more elaborated than a mere translation, but it will in any case satisfy the same previous axioms. For instance, the group parameter $\mu$ may represent the angle of a rotation, or $\mathrm{e}^{\mu}$ may represent the ratio of a homothetic transformation. The above remarks clearly show that it is relevant to associate a Lie symmetry to any master curve (and conversely). Another reason to support the use of the mathematical framework of Lie groups is that any Lie symmetry can have invariants, namely quantities that remain unchanged when the group parameter varies. It will be shown below that this general notion of invariant provides a relevant fundation to support the Dorn and Larson-Miller relationships. However, contrary to the latter which have been formulated in a purely empirical manner, we will try to develop a systematic method to formulate these invariants and combine them to investigate constitutive equations in accordance with the observed symmetries.

The use of Lie groups as a tool to screen the experimental behaviour of some materials and to express their mechanical constitutive laws seems to be for the first time tackled in [14]. The authors provide a methodology for modelling the mechanical behaviour of an acrylic stick solicited at high strain rate. In this strategy, called the inverse method in [9], the final form of the constitutive equation is obtained from a set of experimental data representing the material response for different values of a control parameter, starting from a general functional dependence amongst the control variables, which is fully determined by applying the symmetry conditions.

The aim of the present paper is then to provide an extension of the modelling method previously proposed in [14]. In this previous work, the case of only one generator $\boldsymbol{v}_{1}^{\text {obs }}$ - the shortcut "obs" stands for "observed", recall that vectors are denoted by boldfaced symbols here and in the sequel - and one constitutive equation $\Delta_{1}$ has been investigated. One analyzes here situations for which several generators and several constitutive equations are considered. Accordingly, the algorithm for the definition and classification of observed symmetries will first be presented and will constitute the two first steps of the new method (Step 1 and Step 2). The formulation of the constitutive equations from symmetry conditions will be described in a following step of the algorithm, Step 3. It will be completed with the consideration of invariants of observed symmetries, and the possibility to formulate the constitutive equations as functions of these invariants. In the final step of the method (Step 4), it will be shown that a complete Lie analysis of the constitutive equations can be of great interest to predict new master curves or new charts. Such an analysis can be useful to adjust the mathematical structure of the 
constitutive equations if the predictions are not in good enough agreement with observations. A summary of the entire methodology (steps 1 to 4 ) is represented in the diagram of Fig. 1. In the second part of the paper, the present methodology is exemplified for the $9 \mathrm{Cr} 1 \mathrm{Mo}$ stainless steel submitted to creep tests at different (constant) temperatures and stress levels, extending the behaviour up to rupture.

\section{General Strategy}

As previously stated, the use of Lie groups in combination with experimental responses of a given material to formulate its constitutive law seems to be for the first time tackled in [14]. A general methodology has been provided for modelling the mechanical behaviour of a polymer solicited at high strain rate (acrylic stick at $\dot{\varepsilon} \approx 20 \mathrm{~s}^{-1}$ ). The final form of the constitutive equation is obtained from a set of data representing the material responses at different values of a control parameter. In the present contribution, the methodology is enriched by two novel aspects. The first point is the consideration of several master curves (in different planes) rather than a sole one, resulting in the possibility of predicting new master curves or new theoretical charts by linearly combining their associated Lie symmetries. Thus, the whole methodology will be extended by a predictive aspect, which may be particularly useful to confirm or discriminate the mathematical structure of the identified constitutive equations. The second contribution in the algorithm is the consideration of invariants associated with Lie groups, which offers an alternative to the theoretical formulation of constitutive equations. In the present work, it will be shown that constitutive equations can be formulated by combining invariants, without solving any differential system as done in [14]. As a consequence, the investigation of the behaviour of the studied material becomes more straightforward. Regarding the following section, the generalized methodology is split into four main steps (Fig. 1), each of which being exposed in a separate subsection.

\subsection{Step 1: Formulation of Symmetries From Experimental Data}

The usual strategy of the mechanician or rheologist consists in finding a set of constitutive equations linking a set of observable variables $\boldsymbol{u}=u_{1}, u_{2}, \ldots, u_{n}$ to a set of parameters $\boldsymbol{p}=p_{1}, p_{2}, \ldots, p_{m}$ by analyzing experimental data. The main difference between observable variables and parameters is that parameters are quantities which are controlled during the test, contrary to observable variables. From a physical point of view, the parameters define the solicitation, while the observable variables characterize the mechanical response. For instance, if we consider a uniaxial compression test, we can choose $u_{1}=\sigma$ as the uniaxial Cauchy stress, while 


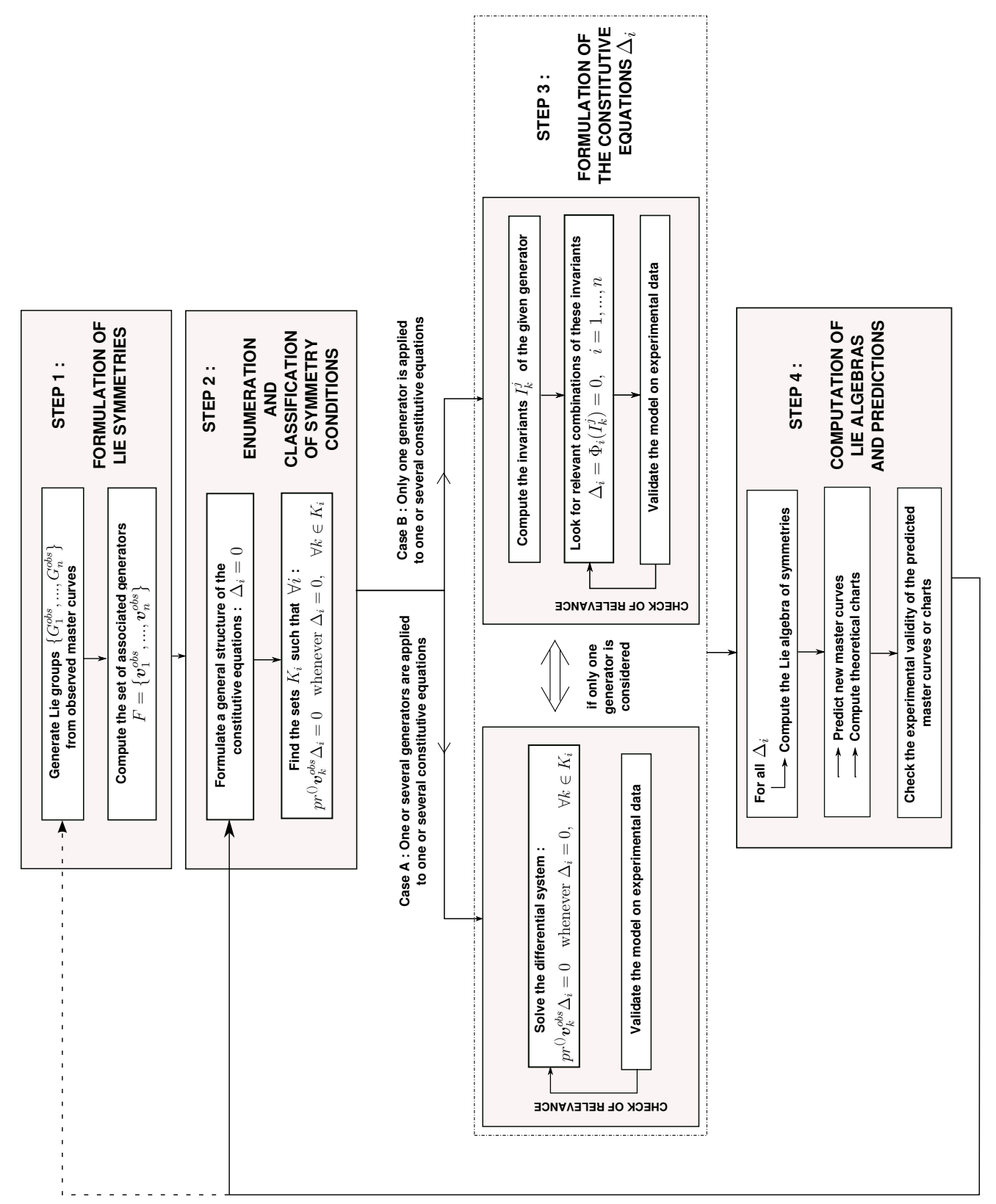

Figure 1. General modelling strategy for materials exhibiting master curves. 
the controlled strain rate $p_{1}=\dot{\varepsilon}$ or the controlled room temperature $p_{2}=T$ are parameters, as well as the time $p_{3}=t$, since its origin may be arbitrarily chosen. Let us now introduce the domain of variations of the variables, denoted here as the following intervals

$$
U_{i}=\left[u_{i}^{\min } ; u_{i}^{\max }\right], \quad i=1, \ldots, n, \quad P_{j}=\left[p_{j}^{\min } ; p_{j}^{\max }\right], \quad j=1, \ldots, m
$$

with $u_{i} \in U_{i}, i=1, \ldots, n$ and $p_{j} \in P_{j}, j=1, \ldots, m$. We shall also denote

$$
\mathcal{U}=U_{1} \times U_{2} \ldots \times U_{n}, \quad \mathcal{P}=P_{1} \times P_{2} \ldots \times P_{m} .
$$

In this work, we focus on the existence of parametric applications $G_{i}^{\text {obs }}(\mu, \boldsymbol{u}, \boldsymbol{p})$ (the superscript "obs" stands for "observed"), $i=1, \ldots, q$, defined as follows

$$
\begin{aligned}
\mathcal{P} \times \mathcal{U} \times \mathbb{R} & \stackrel{G_{i}^{\text {obs }}}{\longrightarrow} \mathcal{P} \times \mathcal{U} \\
{[\boldsymbol{p}, \boldsymbol{u}, \mu] } & \rightarrow[\overline{\boldsymbol{p}}, \overline{\boldsymbol{u}}]=\left[\boldsymbol{\Phi}^{\boldsymbol{p}}(\boldsymbol{p}, \boldsymbol{u}, \mu), \boldsymbol{\Phi}^{\boldsymbol{u}}(\boldsymbol{p}, \boldsymbol{u}, \mu)\right]
\end{aligned}
$$

with $\boldsymbol{\Phi}^{\boldsymbol{p}}=\Phi^{p_{1}}, \ldots, \Phi^{p_{m}}, \boldsymbol{\Phi}^{\boldsymbol{u}}=\Phi^{u_{1}}, \ldots, \Phi^{u_{n}}, \mu$ the parameter, and such that there exists a subset of the experimental data which is "approximatively transformed" into another subset of experimental data. For instance, if we consider the two sets of measurements of the variables $x_{1}$ and $x_{2}$

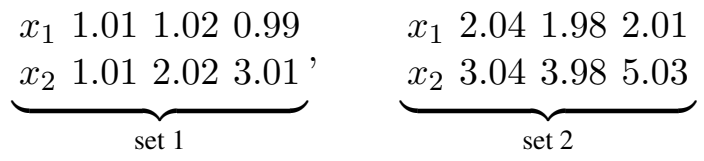

and then the relations

$$
\begin{aligned}
& \bar{x}_{1} \approx x_{1}+\mu \\
& \bar{x}_{2} \approx x_{2}+2 \mu
\end{aligned}
$$

can be viewed as fulfilled for $\mu=0$ (we have the correspondences set $1 \rightarrow$ set 1 , and set $2 \rightarrow$ set 2 ), $\mu=1$ (set $1 \rightarrow$ set 2 ), and $\mu=-1$ ( set $2 \rightarrow$ set 1 ).

Below, we assume that the applications $G_{i}^{\text {obs }}(\mu, \boldsymbol{u}, \boldsymbol{p})$ satisfy the three axioms of a Lie group, that is: existence of a neutral element in the sense of composition

$$
\boldsymbol{\Phi}^{p}(\boldsymbol{p}, \boldsymbol{u}, 0)=\boldsymbol{p}, \quad \boldsymbol{\Phi}^{\boldsymbol{u}}(\boldsymbol{p}, \boldsymbol{u}, 0)=\boldsymbol{u}, \quad \boldsymbol{p} \in \mathcal{P}, \boldsymbol{u} \in \mathcal{U}
$$

reflexivity

$$
\begin{aligned}
{[\bar{p}, \bar{u}] } & =\left[\boldsymbol{\Phi}^{p}(\boldsymbol{p}, \boldsymbol{u}, \mu), \boldsymbol{\Phi}^{\boldsymbol{u}}(\boldsymbol{p}, \boldsymbol{u}, \mu)\right] \\
\Rightarrow \quad[\boldsymbol{p}, \boldsymbol{u}] & =\left[\boldsymbol{\Phi}^{\boldsymbol{p}}(\overline{\boldsymbol{p}}, \overline{\boldsymbol{u}},-\mu), \boldsymbol{\Phi}^{\boldsymbol{u}}(\overline{\boldsymbol{p}}, \overline{\boldsymbol{u}},-\mu)\right], \quad \boldsymbol{p} \in \mathcal{P}, \quad \boldsymbol{u} \in \mathcal{U}
\end{aligned}
$$


and transitivity

$$
\begin{aligned}
{\left[\boldsymbol{p}_{2}, \boldsymbol{u}_{2}\right]=} & {\left[\boldsymbol{\Phi}^{p}\left(\boldsymbol{p}_{1}, \boldsymbol{u}_{1}, \mu_{1}\right), \boldsymbol{\Phi}^{\boldsymbol{u}}\left(\boldsymbol{p}_{1}, \boldsymbol{u}_{1}, \mu_{1}\right)\right] } \\
{\left[\boldsymbol{p}_{3}, \boldsymbol{u}_{3}\right]=} & {\left[\boldsymbol{\Phi}^{\boldsymbol{p}}\left(\boldsymbol{p}_{2}, \boldsymbol{u}_{2}, \mu_{2}\right), \boldsymbol{\Phi}^{\boldsymbol{u}}\left(\boldsymbol{p}_{2}, \boldsymbol{u}_{2}, \mu_{2}\right)\right] } \\
\Rightarrow \quad\left[\boldsymbol{p}_{3}, \boldsymbol{u}_{3}\right]= & {\left[\boldsymbol{\Phi}^{\boldsymbol{p}}\left(\boldsymbol{p}_{1}, \boldsymbol{u}_{1}, \mu_{1}+\mu_{2}\right), \boldsymbol{\Phi}^{\boldsymbol{u}}\left(\boldsymbol{p}_{1}, \boldsymbol{u}_{1}, \mu_{1}+\mu_{2}\right)\right] } \\
& \boldsymbol{p}_{1}, \boldsymbol{p}_{2}, \boldsymbol{p}_{3}, \in \mathcal{P}, \quad \boldsymbol{u}_{1}, \boldsymbol{u}_{2}, \boldsymbol{u}_{3} \in \mathcal{U}
\end{aligned}
$$

In practice, the validity of applications (11) can only be verified for some values of the parameter $\mu$. It is here assumed that (11) are valid in the continuum range of values $\left[\mu_{\min } ; \mu_{\max }\right]$ (with $\mu_{\min }$ and $\mu_{\max }$ the minimal and maximal values of $\mu$ for which (11) is true). It is then possible to introduce a generator $\boldsymbol{v}_{i}^{\text {obs }}$ associated with the Lie group $G_{i}^{\text {obs }}$, defined by

$$
\boldsymbol{v}_{i}^{\mathrm{obs}}=\left.\sum_{k=1}^{m} \frac{\partial \Phi^{p_{k}}}{\partial \mu}\right|_{\mu=0} \frac{\partial}{\partial p_{k}}+\left.\sum_{k=1}^{n} \frac{\partial \Phi^{u_{k}}}{\partial \mu}\right|_{\mu=0} \frac{\partial}{\partial u_{k}}, \quad i=1, \ldots, q .
$$

Summarizing the section, we have here considered $q$ observed symmetries $\boldsymbol{v}_{i}^{\text {obs }}$, $i=1, \ldots, q$.

\subsection{Step 2: Enumeration and Classification of Symmetry Conditions}

The previously-built observed symmetries are next applied to the (currently unknown) postulated constitutive equations. Consequently, let us express those constitutive equations as a set of partial differential equations (PDE) written in the general form

$$
\boldsymbol{\Delta}=\left\{\Delta_{i}=0 ; i=1, \ldots, n\right\}
$$

in which the constitutive model $\Delta_{i}$ may depend on $\boldsymbol{p}$ and all the derivatives of $\boldsymbol{u}$ (including 0 order derivatives, i.e., the quantities $\boldsymbol{u}$ themselves). We assume that there are as many equations as observables, hence the index $i$ varies from 1 to $n$ in (19). No assumption is made for $n$, in the sense that it can be greater or lower than the number of observed symmetries $q$. Equation (19) hides unknown constitutive functions of the variables, which shall be identified by applying the symmetry conditions (see e.g. [14]). In fact, the mathematical structure of equation (19) is defined according to the chosen constitutive framework (e.g. hyperelasticity, viscoelasticity, viscoplasticity). Thus, for a given $\Delta_{i}$, one has to enumerate which of the observed symmetries $\boldsymbol{v}_{k}^{\text {obs }}$ (with $k=1, \ldots, q$ ) will be applied to $\Delta_{i}$ and this amounts defining a subset $K_{i}=\left\{k_{1}, \ldots, k_{m_{i}}\right\}$ of $m_{i}$ indices in $1, \ldots, q$ which 
selects the values of $k$ such that $\boldsymbol{v}_{k}^{\text {obs }}$ is a symmetry of $\Delta_{i}=0$. The range of $K_{i}$, denoted $m_{i}$, corresponds to the total number of symmetries that are applied to $\Delta_{i}$. Consequently, the symmetry conditions write

$$
\begin{aligned}
& \operatorname{pr}^{()} \boldsymbol{v}_{k}^{\text {obs }} \Delta_{1}=0 \quad \text { whenever } \Delta_{1}=0, \quad k \in K_{1} \\
& \operatorname{pr}^{()} \boldsymbol{v}_{k}^{\text {obs }} \Delta_{2}=0 \quad \text { whenever } \Delta_{2}=0, \quad k \in K_{2} \\
& \operatorname{pr}^{()} \boldsymbol{v}_{k}^{\text {obs }} \Delta_{n}=0 \quad \text { whenever } \Delta_{n}=0, \quad k \in K_{n}
\end{aligned}
$$

where the symbols $\operatorname{pr}^{(\kappa)} \boldsymbol{v}_{k}^{\text {obs }}$ or $\operatorname{pr}^{()} \boldsymbol{v}_{k}^{\text {obs }}$ stand for the $\kappa$-th order prolongation $\operatorname{pr}^{()} \boldsymbol{v}_{k}^{\text {obs }}$ is used if the order $\kappa-$ of the vector field $\boldsymbol{v}_{k}^{\text {obs }}$ is not prescribed. The subsets $K_{i}$ are contructed by associating some of the generators $\boldsymbol{v}_{k}^{\text {obs }}$ to $\Delta_{i}$ according to the physical meaning of $\Delta_{i}$. They only have to verify

$$
K_{1} \cup K_{2} \ldots \cup K_{n}=\{1,2, \ldots, q\}
$$

indicating that all the generator $\boldsymbol{v}_{k}^{\text {obs }}$ have been used at least once in equations (20).

\subsection{Step 3: Formulation of the Constitutive Equations $\Delta$}

At this stage, the mathematical expression of the (unknown) constitutive equations can be clarified. For this purpose, two different strategies can be envisaged. The first strategy has been developed in [14] and consists in solving equations (20) with respect to $\Delta$. If a mathematical structure is prescribed for every $\Delta_{i}$, one can expect that the solving of (20) may lead to the final expression of $\Delta_{i}$.

The second strategy consists in calculating the invariants of the generators $\boldsymbol{v}_{k}^{\text {obs }}$ and to find combinations of them that fit the experimental data. Accordingly, let us consider $\boldsymbol{v}^{\text {obs }}$ as any vector field given by (18) and rewritten here as

$$
\boldsymbol{v}^{\mathrm{obs}}=\sum_{k=1}^{m} \phi^{p_{k}} \frac{\partial}{\partial p_{k}}+\sum_{k=1}^{n} \phi^{u_{k}} \frac{\partial}{\partial u_{k}}
$$

with

$$
\phi^{p_{k}}=\left.\frac{\partial \Phi^{p_{k}}}{\partial \mu}\right|_{\mu=0}, \quad \phi^{u_{k}}=\left.\frac{\partial \Phi^{u_{k}}}{\partial \mu}\right|_{\mu=0} .
$$

If $\boldsymbol{v}^{\text {obs }}$ has $s$ non vanishing components amongst $\phi^{\boldsymbol{p}}$ and $\phi^{\boldsymbol{u}}$, it then has $s-1$ invariants $I_{1}, I_{2}, \ldots, I_{s-1}$ given by the solution of the characteristic system

$$
\frac{\mathrm{d} p_{j}}{\phi^{p_{j}}}=\frac{\mathrm{d} u_{i}}{\phi^{u_{i}}}
$$


It is straightforward to show that any function $f\left(I_{1}, I_{2}, \ldots, I_{s-1}\right)$ of the invariants is also invariant under $\boldsymbol{v}^{\text {obs }}$, since

$$
\begin{aligned}
\boldsymbol{v}^{\text {obs }}\left[f\left(I_{1}, \ldots, I_{s-1}\right)\right] & =\sum_{k=1}^{m} \phi^{p_{k}} \frac{\partial f}{\partial p_{k}}+\sum_{k=1}^{n} \phi^{u_{k}} \frac{\partial f}{\partial u_{k}} \\
& =\sum_{k=1}^{m} \phi^{p_{k}} \sum_{l=1}^{s-1} \frac{\partial f}{\partial I_{l}} \frac{\partial I_{l}}{\partial p_{k}}+\sum_{k=1}^{n} \phi^{u_{k}} \sum_{l=1}^{s-1} \frac{\partial f}{\partial I_{l}} \frac{\partial I_{l}}{\partial u_{k}} \\
& =\sum_{l=1}^{s-1} \frac{\partial f}{\partial I_{l}} \underbrace{\left[\sum_{k=1}^{m} \phi^{p_{k}} \frac{\partial I_{l}}{\partial p_{k}}+\sum_{k=1}^{n} \phi^{u_{k}} \frac{\partial I_{l}}{\partial u_{k}}\right]}_{\boldsymbol{v}^{\text {obs }}\left(I_{l}\right)=0}=0 .
\end{aligned}
$$

Hence, the constitutive equation $\Delta_{i}$ can be expressed by combining the invariants $I_{1}, I_{2}, \ldots, I_{s-1}$, provided it fits the experimental data. The main limitation of this methodology is that it cannot be applied if more than one generator is considered: indeed, if we denote $\boldsymbol{v}^{\text {obs }}$ and $\boldsymbol{w}^{\text {obs }}$ two generators having invariants $I_{1}, \ldots, I_{s-1}$ and $J_{1}, \ldots, J_{r-1}$ respectively, and a new function $f$ of those combined invariants, we then have

$$
\begin{aligned}
\boldsymbol{v}^{\mathrm{obs}}\left[f\left(I_{1}, \ldots, I_{s-1}, J_{1}, \ldots, J_{r-1}\right)\right] & =\sum_{l}^{r-1} \frac{\partial f}{\partial J_{l}} \boldsymbol{v}^{\mathrm{obs}}\left(J_{l}\right) \neq 0 \\
\boldsymbol{w}^{\mathrm{obs}}\left[f\left(I_{1}, \ldots, I_{s-1}, J_{1}, \ldots, J_{r-1}\right)\right] & =\sum_{l}^{s-1} \frac{\partial f}{\partial I_{l}} \boldsymbol{w}^{\mathrm{obs}}\left(I_{l}\right) \neq 0
\end{aligned}
$$

which shows that a general combination of the two sets of invariants is not a priori invariant under the experimental symmetry groups. The generalization to any number of generators greater than two is obvious and consequently, the derivation of a constitutive equation $\Delta_{i}$ written as a function of the invariants of $\boldsymbol{v}_{k}^{\text {obs }}, k \in K_{i}$, is only possible if $m_{i}=1$.

\subsection{Step 4: Computation of the Lie Algebras and Predictions}

At this stage, it is assumed that $\Delta$ is completely determined by the previous step, and that it is coherent with the experimental data. The present step of the algorithm consists in a complete Lie analysis of $\Delta$. More precisely, for each $\Delta_{i}$, one computes the Lie algebra

$$
A_{i}=\boldsymbol{v}_{i}^{1}, \boldsymbol{v}_{i}^{2}, \ldots, \boldsymbol{v}_{i}^{n_{i}}
$$


containing the $n_{i}$ vector fields that can generate (by linear combination) any Lie symmetry of $\Delta_{i}$. Obviously, the vector fields $\boldsymbol{v}_{k}^{\text {obs }}, k \in K_{i}$, have to be generated by the vector fields (27), that is

for all $\boldsymbol{v}_{k}^{\text {obs }}, k \in K_{i}$, and there exists $\boldsymbol{\alpha} \in \mathbb{R}^{n_{i}}$ such that $\boldsymbol{v}_{k}^{\text {obs }}=\sum_{j=1}^{n_{i}} \alpha_{j} \boldsymbol{v}_{i}^{j}$.

The interest of calculating the Lie algebra is twofold. First, it is possible to predict new master curves valid in some graphical representation. Indeed, one can consider suitable linear combinations of the vector fields $\boldsymbol{v}_{i}^{j}$ for which one or several wellchosen components vanish. It is then possible to carry out new experiments which shall confirm or invalidate the predicted master curve. If the master curve is not observed, then the mathematical expression of $\Delta_{i}$ (or even, the expression of $\boldsymbol{v}_{k}^{\text {obs }}$ ) has to be adjusted, such that every master curve predicted by its Lie algebra is in coherence with the experimental data.

Among the set of all combinations of $\boldsymbol{v}_{i}^{j}$, we can also focus on vector fields for which (i) the component of a given observable $u_{j}$ vanishes and (ii) the other components do not depend on $u_{j}$

$$
\boldsymbol{v}=\sum_{k=1}^{m} \phi^{p_{k}}\left(\boldsymbol{p}, \boldsymbol{u}^{-j}\right) \frac{\partial}{\partial p_{k}}+\sum_{k=1, k \neq j}^{n} \phi^{u_{k}}\left(\boldsymbol{p}, \boldsymbol{u}^{-j}\right) \frac{\partial}{\partial u_{k}}
$$

with $\boldsymbol{u}^{-j}=u_{1}, \ldots, u_{j-1}, u_{j+1}, \ldots, u_{n}$. The flow of such a vector field takes the particular form

$$
\begin{aligned}
\overline{\boldsymbol{p}} & =\overline{\boldsymbol{p}}\left(\boldsymbol{p}, \boldsymbol{u}^{-j}, \mu\right) \\
\overline{\boldsymbol{u}}^{-j} & =\overline{\boldsymbol{u}}^{-j}\left(\boldsymbol{p}, \boldsymbol{u}^{-j}, \mu\right) \\
\bar{u}_{j} & =u_{j} .
\end{aligned}
$$

Hence, eliminating the parameter $\mu$ allows a priori to determine the equations of the "iso- $u_{j}$ " curves in the space $\mathcal{P} \times \mathcal{U}^{-j}$, with

$$
\mathcal{U}^{-j}=U_{1} \times \ldots U_{j-1} \times U_{j+1} \ldots \times U_{n} .
$$

This kind of chart may be an interesting tool to extrapolate experimental data when the value of some parameter $p_{k}$ cannot be reached in the experimental setting.

Summarizing this section, the step 4 of the algorithm is in fact a phase of prediction and validation, since the results induced by the Lie algebra have to be validated by experiments. The entire methodology (steps 1 to 4 ) is represented in the diagram in Fig. 1. Let us now apply the proposed strategy to creep strain phenomena, which are known to be highly dependent on the applied stress and temperature. 


\section{Application to the 9Cr1Mo Creep: Observed Symmetries}

Ferritic-martensitic steels have been extensively studied during the last 30 years, due to the need to increase steam conditions (temperature and pressure). According to the authors of [26], they are commonly used in supercritical and ultrasupercritical plants with steam conditions close to $903 \mathrm{~K}$ and $30 \mathrm{MPa}$. Ferritic-martensitic steels with $\mathrm{Cr}$ contents ranging from $9 \%$ to $12 \%$ in weight are also being considered in different applications in various generations of advanced nuclear power plant designs, such as pressure vessels, tubing and piping, and fuel cladding. Those heat-resistant steels typically contain $0.1 \%$ to $0.2 \%$ carbon and small additions of refractory metals such as Mo, $\mathrm{V}$, and $\mathrm{W}$. They constitute an attractive substitute for austenitic stainless steels due to their reduced cost, lower thermal expansion coefficient, and better radiation stability combined with adequate corrosion resistance (see [26] and the references therein).

Here We rely on the experimental data of [24], obtained for the above-mentioned 9Cr1Mo alloy, when submitted to creep tests carried out at different constant temperatures and different constant stress levels. In the present case, the observable variables are the strain rate $u_{1}=\dot{\varepsilon}$ and the time to rupture $u_{2}=t_{R}$, while the control variables are the applied stress $p_{1}=\sigma$, the absolute temperature $p_{2}=T$, and the time $p_{3}=t$. Let us notice that applying a function to a physical quantity means that the function is applied to the measure of the quantity in its own unit: for instance, $\log \sigma$ stands for $\log \left(\sigma / \sigma_{0}\right)$ with $\sigma_{0}=1 \mathrm{~Pa}$ if $\sigma$ is written in Pa. Below, the following units will be considered: hours (h) for the time $t$, megaPascal (MPa) for the stress, Kelvin (K) for the temperature, percents $(\%)$ for the strain.

\subsection{Step 1}

\subsubsection{Rupture Curves}

In this section, isothermal rupture curves at different temperatures are first considered in the logarithmic plane. If we consider only "simple" geometrical mappings such as translations or homothetic mappings, it can be seen that a master curve can be obtained by shifting the curves along the $\log t_{R}$ and $\log \sigma$ axis, as shown in Fig. 2. From a mathematical point of view, one then looks for the following geometrical mapping

$$
\begin{aligned}
\bar{T} & =T+\mu \\
\log \bar{t}_{R} & =a_{1}(\mu)+\log t_{R} \\
\log \bar{\sigma} & =b_{1}(\mu)+\log \sigma
\end{aligned}
$$




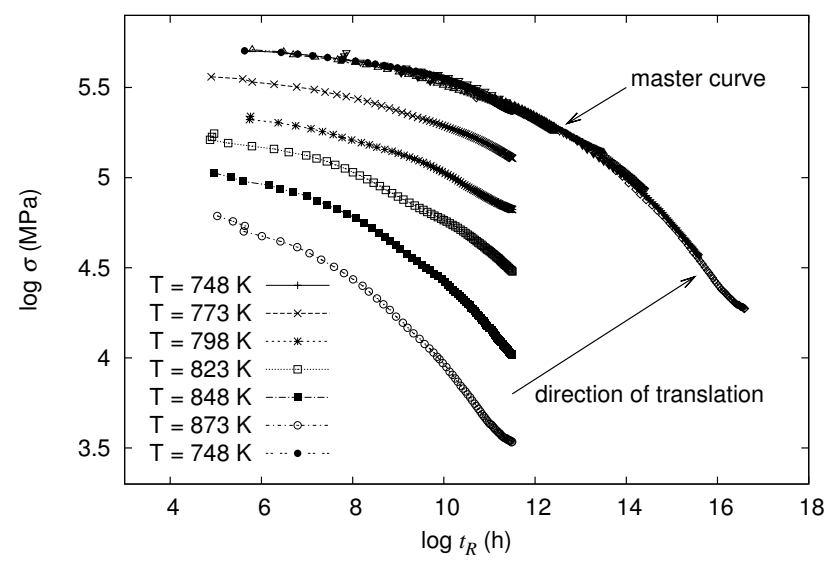

Figure 2. Experimental master curve at the reference temperature $\bar{T}=748 \mathrm{~K}$ obtained by shifting the curves along the $\log t_{R}$ and $\log \sigma$ axis.

with $a_{1}(\mu)$ and $b_{1}(\mu)$ the shift factors along the $\log t_{R}$ and $\log \sigma$ axis respectively. If the (arbitrary) chosen value of $\bar{T}$ is $748 \mathrm{~K}$, then $a_{1}(\mu)$ and $b_{1}(\mu)$ correspond to the shift required to move the curve at $T$ to make it coincide with those at $\bar{T}$, if $\mu$ is defined by $\bar{T}=T+\mu$. The values of $\mu, a_{1}(\mu)$ and $b_{1}(\mu)$ leading to the construction of the master curve of Fig. 2 are given in Table 2.

Table 2. Values of the shifts $a_{1}(\mu)$ and $b_{1}(\mu)$ to superpose a given curve at $T$ on the reference response at $\bar{T}=748 \mathrm{~K}, \mu$ being defined by $\bar{T}=T+\mu$.

\begin{tabular}{c|cccccc}
$T(\mathrm{~K})$ & 748 & 773 & 798 & 823 & 848 & 873 \\
\hline$\mu$ & 0 & -25 & -50 & -75 & -100 & -125 \\
$a_{1}(\mu)$ & 0 & 0.9 & 2.0 & 2.9 & 4.1 & 5.1 \\
$b_{1}(\mu)$ & 0 & 0.15 & 0.32 & 0.45 & 0.55 & 0.74
\end{tabular}

If we assume that equations(32) define a Lie group, then the three axioms presented in Section 2.1 have to be fulfilled. It can be readily shown that they yield the following conditions to be satisfied by functions $a_{1}(\mu)$ and $b_{1}(\mu)$

$$
\begin{aligned}
a_{1}(0) & =0 & b_{1}(0) & =0 \\
a_{1}(-\mu) & =-a_{1}(\mu) & b_{1}(-\mu) & =-b_{1}(\mu) \\
a_{1}\left(\mu_{1}+\mu_{2}\right) & =a_{1}\left(\mu_{1}\right)+a_{1}\left(\mu_{2}\right), & b_{1}\left(\mu_{1}+\mu_{2}\right) & =b_{1}\left(\mu_{1}\right)+b_{1}\left(\mu_{2}\right)
\end{aligned}
$$


for all $\mu, \mu_{1}, \mu_{2}$ in $\mathbb{R}$. From equations (33), one can infer the linearity of $a_{1}(\mu)$ and $b_{1}(\mu)$ with respect to $\mu$

$$
a_{1}(\mu)=\alpha \mu, \quad b_{1}(\mu)=\beta \mu
$$

where $\alpha$ and $\beta$ are true constants. Those last relations are in great accordance with the experimental values of the shift factors, since linear fits of $a_{1}(\mu)$ and $b_{1}(\mu)$ exhibit good correlations. The two fits give the following values of the coefficients

$$
\alpha=-0.0403636, \quad \beta=-0.00584727
$$

that will be used here and in all subsequent developments. Hence, if one inserts (34) into (32), the following observed Lie group $G_{1}^{\text {obs }}$ is obtained

$$
\bar{T}=T+\mu, \quad \bar{t}_{R}=\mathrm{e}^{\alpha \mu} t_{R}, \quad \bar{\sigma}=\mathrm{e}^{\beta \mu} \sigma
$$

and maps an experimental curve into another one. Of course, this Lie group is only valid for the explored values of $T$, but its validity is presently assumed for all $T$ between the extremal values $748 \mathrm{~K}$ and $923 \mathrm{~K}$. The components of the generator $\boldsymbol{v}_{1}^{\text {obs }}$ associated with $G_{1}^{\text {obs }}$ are given by the derivation of equations (36) with respect to $\mu$ at $\mu=0$, viz

$$
\boldsymbol{v}_{1}^{\mathrm{obs}}=\alpha t_{R} \frac{\partial}{\partial t_{R}}+\beta \sigma \frac{\partial}{\partial \sigma}+\frac{\partial}{\partial T} .
$$

Thereby, and as summary, the infinitesimal generator allowing to shift the measured rupture curve for a given temperature to another neighboring temperature in the stress-rupture time plane has been obtained. The Lie group generated by this vector field allows to shift all rupture responses in the same plane to the response obtained for a chosen reference temperature while the curve associated with this response is designated as the master curve.

\subsubsection{Creep Curves}

A similar strategy may be applied to the "isostrain" creep curves in the logarithmic plane $\log \sigma$ vs $\log t$. As previously, one shall search the transformation rules

$$
\begin{aligned}
\bar{\varepsilon} & =\mathrm{e}^{\mu} \varepsilon \\
\log \bar{t} & =a_{2}(\mu)+\log t \\
\log \bar{\sigma} & =b_{2}(\mu)+\log \sigma
\end{aligned}
$$

mapping a curve at $\varepsilon$ to a reference curve at $\bar{\varepsilon}=5 \%$ (observe that the chosen value of the reference strain is arbitrary). The corresponding values of the shifts $a_{2}(\mu)$ 
Table 3. Values of the shifts $a_{2}(\mu)$ and $b_{2}(\mu)$ to superpose a given curve at $\varepsilon$ on the reference response at $\bar{\varepsilon}=5 \%, \mu$ being defined by $\bar{\varepsilon}=\mathrm{e}^{\mu} \varepsilon$.

\begin{tabular}{c|cccccc}
$\varepsilon(\%)$ & 5.0 & 2.0 & 1.0 & 0.5 & 0.2 & 0.1 \\
\hline$\mu$ & 0 & 0.92 & 1.61 & 2.30 & 3.22 & 3.91 \\
$a_{2}(\mu)$ & 0 & 1.15 & 2.18 & 3.33 & 4.71 & 6.78 \\
$b_{2}(\mu)$ & 0 & -0.023 & -0.032 & -0.046 & -0.053 & -0.062
\end{tabular}

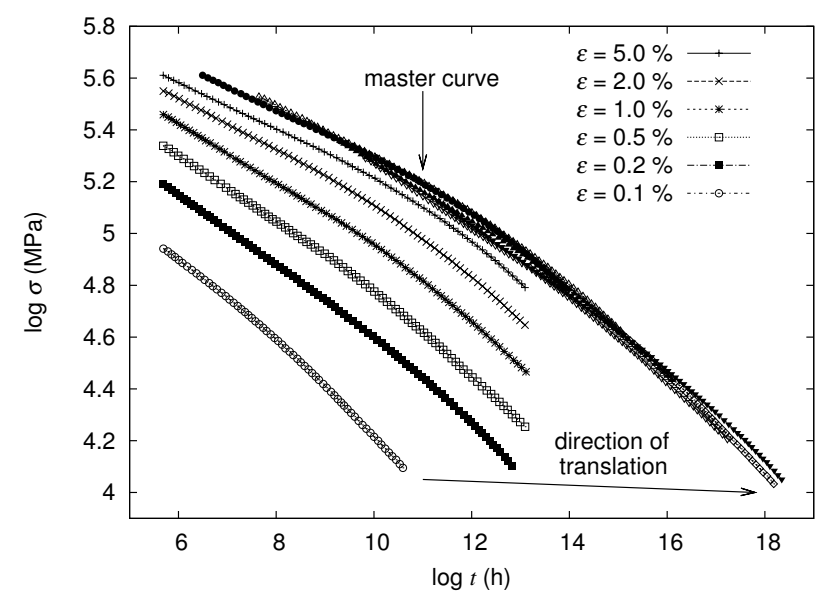

Figure 3. Master curve obtained at the reference strain $\bar{\varepsilon}=5.0 \%$ by shifting the different responses along the $\log t$ and $\log \sigma$ axis.

and $b_{2}(\mu)$ are listed in Table 3 and the obtained master curve is shown in Fig. 3. The axioms of Lie symmetries lead as in Section 3.1.1.to the linearity of $a_{2}(\mu)$ and $b_{2}(\mu)$ with respect to $\mu$

$$
a_{2}(\mu)=\gamma \mu, \quad b_{2}(\mu)=\delta \mu
$$

with $\gamma$ and $\delta$ new constants given by linear fit

$$
\gamma=1.57167, \quad \delta=-0.0172415
$$

A second Lie group $G_{2}^{\text {obs }}$ is then obtained by inserting (39) into (38)

$$
\bar{\varepsilon}=\mathrm{e}^{\mu} \varepsilon, \quad \bar{t}=\mathrm{e}^{\gamma \mu} t, \quad \bar{\sigma}=\mathrm{e}^{\delta \mu} \sigma .
$$

The generator $\boldsymbol{v}_{2}^{\mathrm{obs}}$ of $G_{2}^{\mathrm{obs}}$ is given by

$$
\boldsymbol{v}_{2}^{\mathrm{obs}}=\gamma t \frac{\partial}{\partial t}+\delta \sigma \frac{\partial}{\partial \sigma}+\varepsilon \frac{\partial}{\partial \varepsilon} .
$$


The prolongations of the vector fields $\boldsymbol{v}_{1}^{\text {obs }}$ and $\boldsymbol{v}_{2}^{\text {obs }}$ account for the component with respect to $\dot{\varepsilon}$ and shall prove useful in the sequel. They can be computed with the prolongation formulae (see e.g. [15])

$$
\begin{aligned}
& \operatorname{pr}^{(1)} \boldsymbol{v}_{1}^{\text {obs }}=\alpha t_{R} \frac{\partial}{\partial t_{R}}+\beta \sigma \frac{\partial}{\partial \sigma}+\frac{\partial}{\partial T} \\
& \operatorname{pr}^{(1)} \boldsymbol{v}_{2}^{\text {obs }}=\gamma t \frac{\partial}{\partial t}+\delta \sigma \frac{\partial}{\partial \sigma}+\varepsilon \frac{\partial}{\partial \varepsilon}+\dot{\varepsilon}(1-\gamma) \frac{\partial}{\partial \dot{\varepsilon}} .
\end{aligned}
$$

It is now possible to search for constitutive equations of the studied alloy.

\subsection{Step 2}

At this stage, recall that two generators ( $\boldsymbol{v}_{1}^{\mathrm{obs}}$ and $\boldsymbol{v}_{2}^{\mathrm{obs}}$ ) have been identified from the experimental data set (step 1). Following the strategy of Section 2, we now look for a set of constitutive equations that can capture the creep behaviour of the material. As written in the introduction, one classically admits that the set of constitutive equations can be split into two relations revealing two different physical informations. The first one expresses the time to rupture $t_{R}$ versus the loading parameters ( $T$ and $\sigma$ in the present case) while the second one gives the evolution of the strain rate $\dot{\varepsilon}$ as a function of $t, \sigma, \varepsilon$, and $T$

$$
\begin{aligned}
& \Delta_{1}=t_{R}-f(\sigma, T)=0 \\
& \Delta_{2}=\dot{\varepsilon}-h(t, \sigma, \varepsilon, T)=0 .
\end{aligned}
$$

In those two equations, $h$ and $f$ are unknown functions that will be further determined. As concerns the classification of symmetries, it seems reasonable to apply the symmetry condition $\boldsymbol{v}_{1}^{\text {obs }}$ to $\Delta_{1}$ and $\boldsymbol{v}_{2}^{\text {obs }}$ to $\Delta_{2}$. Indeed, the generator $\boldsymbol{v}_{1}^{\text {obs }}$ ( $\boldsymbol{v}_{2}^{\text {obs }}$ respectively) has been built by only considering rupture curves (creep curves respectively). The satisfaction of those symmetry conditions means that each constitutive law (creep and rupture) keeps the same form and remains valid when the considered parameter changes. Finally, the complete set of symmetry conditions writes

$$
\begin{aligned}
& \operatorname{pr}^{(1)} \boldsymbol{v}_{1}^{\text {obs }} \Delta_{1}=0, \quad \text { whenever } \Delta_{1}=0 \\
& \operatorname{pr}^{(1)} \boldsymbol{v}_{2}^{\text {obs }} \Delta_{2}=0, \quad \text { whenever } \Delta_{2}=0
\end{aligned}
$$

and it is associated with the following sets $K_{1}=\{1\}$ and $K_{2}=\{2\}$. The next step consists in writing the constitutive models in terms of invariants. 


\subsection{Step 3}

As $m_{1}=1$ and $m_{2}=1$, the two methodologies described in Section 2.3 can be applied. The first methodology (direct solving of the symmetry conditions) has already been applied in [14] and here we shall consider the strategy involving a combination of invariants (case B in Fig. 1), in the spirit of the extrapolation methods of the literature (see the review article [22] and references therein). Thus, one looks for two new functions $\Sigma_{1}\left(I_{1}, I_{2}\right)$ and $\Sigma_{2}\left(J_{1}, J_{2}, J_{3}\right)$ such that

$$
\begin{aligned}
\Sigma_{1}\left(I_{1}, I_{2}\right) & =0 \Leftrightarrow \Delta_{1}=0 \\
\Sigma_{2}\left(J_{1}, J_{2}, J_{3}\right) & =0 \Leftrightarrow \Delta_{2}=0
\end{aligned}
$$

with $I_{1}, I_{2}$ the invariants of $\boldsymbol{v}_{1}^{\text {obs }}$ given by the solution of the system (24)

$$
\frac{\mathrm{d} t_{R}}{\alpha t_{R}}=\frac{\mathrm{d} \sigma}{\beta \sigma}=\frac{\mathrm{d} T}{T}
$$

and $J_{1}, J_{2}, J_{3}$ the invariants of $\boldsymbol{v}_{2}^{\text {obs }}$ given by the solution of the same characteristic system

$$
\frac{\mathrm{d} t}{\gamma t}=\frac{\mathrm{d} \sigma}{\delta \sigma}=\frac{\mathrm{d} \varepsilon}{\varepsilon}=\frac{\mathrm{d} \dot{\varepsilon}}{(1-\gamma) \dot{\varepsilon}} .
$$

Solving (50) and (51) gives the following invariants for rupture

$$
I_{1}=\log t_{R}-\alpha T, \quad I_{2}=\log \sigma-\beta T
$$

and creep

$$
J_{1}=\log t-\gamma \log \varepsilon, \quad J_{2}=\log \sigma-\delta \log \varepsilon, \quad J_{3}=\log \dot{\varepsilon}-(1-\gamma) \log \varepsilon .
$$

Considering rupture, we suggest a relation having the form

$$
\log t_{R}=\frac{F_{1}(\sigma)}{T}+F_{2}(\sigma)
$$

We have decided to keep the dependence of the slope $F_{1}$ with respect to $\sigma$, since the very low number of experimental points does not allow to assume a constant slope. To follow as closely as possible the mathematical structure of (54), the following expression is proposed

$$
\Sigma_{1}\left(I_{1}, I_{2}\right)=\frac{K_{1}}{I_{2}}+K_{2} I_{1}+K_{3} I_{2}=0
$$

in which $K_{1}, K_{2}, K_{3}$ are constants, which warrants a hyperbolic dependence with respect to $T$. Equation (55) leads to

$$
\log t_{R}=\frac{a}{\log \sigma-\beta T}+b \log \sigma+(\alpha-b \beta) T
$$


with $a=-K_{1} / K_{2}$ and $b=-K_{3} / K_{2}$ new constants. Equation (56) shows that a linear term in $T$ appears by combining the invariants $I_{1}$ and $I_{2}$, giving a relation which slightly differs from the Larson-Miller or Dorn relationships. However, this linear term is necessary to fullfill the symmetry condition (46) that follows from the experimental data set. A good validation of equation (56) with the experimental data presented in [24] can be observed (the coefficients $a$ and $b$ are adjusted). As to the creep strain rate, we introduce in accordance with the "convenient creep laws" of [24] the power function

$$
\Sigma_{2}\left(J_{1}, J_{2}, J_{3}\right)=\mathrm{e}^{J_{3}}-A \mathrm{e}^{c J_{1}} \mathrm{e}^{d J_{2}}=\dot{\varepsilon} \varepsilon^{-(1-\gamma)}-A t^{c} \varepsilon^{-c \gamma} \sigma^{d} \varepsilon^{-d \delta}=0
$$

with $A, c$ and $d$ some constants, which leads to

$$
\dot{\varepsilon}=A t^{c} \sigma^{d} \varepsilon^{1-\gamma-c \gamma-d \delta} .
$$

It can be easily shown that the symmetry conditions (46) and (47) are fulfilled. If we denote by (i) $\varepsilon(t=0, \sigma, T)=\varepsilon_{0}(\sigma, T)$ the initial value of the strain $\varepsilon$ and (ii) the exponent $\nu=\gamma+c \gamma+d \delta$, then the resolution of equation (58) allows to write

$$
\varepsilon(t)=(1+c)^{-1 / \nu}\left[(1+c) \varepsilon_{0}(\sigma, T)^{\nu}+A \nu t^{c+1} \sigma^{d}\right]^{1 / \nu} .
$$

If the temperature $T$ and the strain $\varepsilon$ are assigned fixed values, and if we assume that the initial strain can be written in terms of a Hookean relation

$$
\varepsilon_{0}(\sigma, T)=\frac{\sigma}{E(T)}
$$

with $E(T)$ the Young modulus at $T$, then the "isostrain" curves may be obtained by expressing the time $t(\sigma)$ from equation (59), viz

$$
t(\sigma)=\left[\frac{(c+1)\left(\varepsilon^{\nu}-\sigma^{\nu} E(T)^{-\nu}\right)}{A \nu \sigma^{d}}\right]^{1 /(1+c)} .
$$

The inverse functional dependence $\sigma(t)$ can be numerically calculated. The validation of the model $\log \sigma$ vs $\log t$ can then be carried out through equation (61) and we have observed that the model and the experimental data are in good agreement (the coefficients $E(T), A$ and $c, d$ are adjusted).

\subsection{Step 4}

To summarize the previous sections, the following constitutive equations have been obtained as representative of the creep and rupture behaviours of the considered $9 \mathrm{Cr} 1 \mathrm{Mo}$ stainless steel

$$
\begin{aligned}
\dot{\varepsilon} & =A t^{c} \sigma^{d} \varepsilon^{1-\gamma-c \gamma-d \delta} \quad \text { if } \quad t \leq t_{R} \\
t_{R} & =\sigma^{b} \mathrm{e}^{\frac{a}{\log \sigma-\beta T}+(\alpha-b \beta) T} .
\end{aligned}
$$


Those formulations of the constitutive laws are compatible with the observed master curves. We can now proceed to the Lie analysis of these constitutive equations.

\subsubsection{Lie Algebra of the Creep Model}

The Lie algebra of the creep model is computed following the methodology described in [15]. It contains 4 generators $\boldsymbol{v}_{2}^{i}, i=1, \ldots, 4$ with prolongations given by:

$$
\begin{aligned}
& \operatorname{pr}^{(1)} \boldsymbol{v}_{2}^{1}=\sigma^{d} t^{-c} \frac{\partial}{\partial t}+c \dot{\varepsilon} \sigma^{d} t^{-c-1} \frac{\partial}{\partial \dot{\varepsilon}} \\
& \operatorname{pr}^{(1)} \boldsymbol{v}_{2}^{2}=\sigma^{d} \varepsilon^{1-\nu} \frac{\partial}{\partial \varepsilon}+(1-\nu) \sigma^{d} \varepsilon^{-\nu} \dot{\varepsilon} \frac{\partial}{\partial \dot{\varepsilon}} \\
& \operatorname{pr}^{(1)} \boldsymbol{v}_{2}^{3}=t \frac{\partial}{\partial t}+\left(\frac{c+1}{\nu}\right) \varepsilon \frac{\partial}{\partial \varepsilon}+\left(\frac{c+1-\nu}{\nu}\right) \dot{\varepsilon} \frac{\partial}{\partial \dot{\varepsilon}} \\
& \operatorname{pr}^{(1)} \boldsymbol{v}_{2}^{4}=t \frac{\partial}{\partial t}-\left(\frac{c+1}{d}\right) \sigma \frac{\partial}{\partial \sigma}-\dot{\varepsilon} \frac{\partial}{\partial \dot{\varepsilon}}
\end{aligned}
$$

in which we recall that $\nu=\gamma+c \gamma+d \delta$. The commutation table of this Lie algebra is given in Table 4. In order to show the practical interest of this table, let

Table 4. Commutator table of the Lie algebra of equation (62).

\begin{tabular}{c|cccc} 
& $\boldsymbol{v}_{2}^{1}$ & $\boldsymbol{v}_{2}^{2}$ & $\boldsymbol{v}_{2}^{3}$ & $\boldsymbol{v}_{2}^{4}$ \\
\hline $\boldsymbol{v}_{2}^{1}$ & 0 & 0 & $(c+1) \boldsymbol{v}_{2}^{1}$ & $2(c+1) \boldsymbol{v}_{2}^{1}$ \\
$\boldsymbol{v}_{2}^{2}$ & 0 & 0 & $(c+1) \boldsymbol{v}_{2}^{2}$ & $(c+1) \boldsymbol{v}_{2}^{2}$ \\
$\boldsymbol{v}_{2}^{3}$ & $-(c+1) \boldsymbol{v}_{2}^{1}$ & $-(c+1) \boldsymbol{v}_{2}^{2}$ & 0 & 0 \\
$\boldsymbol{v}_{2}^{4}$ & $-2(c+1) \boldsymbol{v}_{2}^{1}$ & $-(c+1) \boldsymbol{v}_{2}^{2}$ & 0 & 0
\end{tabular}

us consider the particular combination of the calculated generators

$$
\begin{aligned}
\frac{\nu}{c+1}\left(\operatorname{pr}^{(1)} \boldsymbol{v}_{2}^{3}+\left(\frac{c+1-\nu}{\nu}\right) \operatorname{pr}^{(1)} \boldsymbol{v}_{2}^{4}\right) & \\
& =t \frac{\partial}{\partial t}+\varepsilon \frac{\partial}{\partial \varepsilon}-\left(\frac{c+1-\nu}{d}\right) \sigma \frac{\partial}{\partial \sigma}
\end{aligned}
$$

which has no component with respect to $\dot{\varepsilon}$ and which is obviously a Lie symmetry of (62). The flow of this generator is given by

$$
\begin{aligned}
\bar{t} & =\mathrm{e}^{\mu} t \\
\bar{\sigma} & =\mathrm{e}^{-\left(\frac{c+1-\nu}{d}\right) \mu} \sigma \\
\bar{\varepsilon} & =\mathrm{e}^{\mu} \varepsilon
\end{aligned}
$$


or, by taking the logarithm

$$
\begin{aligned}
\log \bar{t} & =\mu+\log t \\
\log \bar{\sigma} & =-\left(\frac{c+1-\nu}{d}\right) \mu+\log \sigma \\
\log \bar{\varepsilon} & =\mu+\log \varepsilon
\end{aligned}
$$

This last set of relations is of great interest since it describes a master curve in the $\log \varepsilon$ vs $\log t$ representation. More precisely, the curve $\log \bar{\varepsilon}$ vs $\log \bar{t}$ obtained for a stress level $\bar{\sigma}$ is linked to the curve obtained for $\sigma$ by a translation of vector $(\mu, \mu)$, the shift being defined by $\mu=\frac{d}{c+1-\nu} \log (\sigma / \bar{\sigma})$. This master curve has not been validated because too few creep curves $\varepsilon(t)$ at different stress levels have been found. Consequently, an experimental investigation should be of great interest to validate or discard this master curve.

\subsubsection{Lie Algebra of Equation (63)}

The Lie algebra has two generators $\boldsymbol{v}_{1}^{i}, i=1,2$, given by

$$
\begin{aligned}
& \boldsymbol{v}_{1}^{1}=t_{R}\left(\frac{b(\log \sigma-\beta T)^{2}-a}{\log \sigma-\beta T}\right) \frac{\partial}{\partial t_{R}}+\sigma(\log \sigma-\beta T) \frac{\partial}{\partial \sigma} \\
& \boldsymbol{v}_{1}^{2}=t_{R}\left(\frac{(\alpha-b \beta)(\log \sigma-\beta T)^{2}+a \beta}{\log \sigma-\beta T}\right) \frac{\partial}{\partial t_{R}}+(\log \sigma-\beta T) \frac{\partial}{\partial T}
\end{aligned}
$$

and its commutator table is given in Table 5. If we denote by $\phi^{1, t_{R}}$ and $\phi^{2, t_{R}}$ the

Table 5. Commutator table of the Lie algebra of equation (63).

\begin{tabular}{c|cc} 
& $\boldsymbol{v}_{1}^{1}$ & $\boldsymbol{v}_{1}^{2}$ \\
\hline $\boldsymbol{v}_{1}^{1}$ & 0 & $\beta \boldsymbol{v}_{1}^{1}+\boldsymbol{v}_{1}^{2}$ \\
$\boldsymbol{v}_{1}^{2}$ & $-\beta \boldsymbol{v}_{1}^{1}-\boldsymbol{v}_{1}^{2}$ & 0
\end{tabular}

component in $t_{R}$ of $\boldsymbol{v}_{1}^{1}$ and $\boldsymbol{v}_{1}^{2}$ respectively, then the following local combination

$$
\begin{aligned}
\boldsymbol{v} & =\frac{\phi^{2, t_{R}} \boldsymbol{v}_{1}^{1}-\phi^{1, t_{R}} \boldsymbol{v}_{1}^{2}}{t_{R}} \\
& =\sigma\left[a \beta+(\alpha-b \beta)(\log \sigma-\beta T)^{2}\right] \frac{\partial}{\partial \sigma}\left(a-b(\log \sigma-\beta T)^{2}\right) \frac{\partial}{\partial T}
\end{aligned}
$$

is a symmetry of equation (63) having no component in $t_{R}$ and whose components in $\sigma$ and $T$ do not depend on $t_{R}$. Consequently, $\boldsymbol{v}$ has the form of equation (29), 
and the flow of this vector field is given by the solution of the following system of differential equations

$$
\begin{aligned}
& \frac{\mathrm{d} \bar{\sigma}}{\mathrm{d} \mu}=\bar{\sigma}\left[a \beta+(\alpha-b \beta)(\log \bar{\sigma}-\beta \bar{T})^{2}\right] \\
& \frac{\mathrm{d} \bar{T}}{\mathrm{~d} \mu}=a-b(\log \bar{\sigma}-\beta \bar{T})^{2}
\end{aligned}
$$

with the initial conditions

$$
\bar{\sigma}(0)=\sigma, \quad \bar{T}(0)=T
$$

and the equality $\bar{t}_{R}=t_{R}$. Consequently, the numerical solution of (74) and (75) provides a parametric representation $(T(\mu), \sigma(\mu))$ of the "iso- $t_{R}$ " curves in the $(T, \sigma)$ plane. This kind of theoretical chart is of high practical interest, as it allows to extrapolate the rupture time of a specimen at any stress level or any temperature, provided that the rupture time is known for a given value of $\sigma$ and $T$.

\section{Discussion and Concluding Remarks}

Lie symmetries can be considered as a powerful tool in the Mechanics of Materials, in order to construct the constitutive law of a given material from experimental results, as a functional relation between control variables and additional parameters. Thereby, the symmetry method is exploited to solve an inverse problem, assuming the measured data have a structure akin to a Lie group, when considering a continuous range of variation of the adopted parameters. Recall that two main class of approaches have arisen in the literature, which will be referred to as the direct and the inverse problem or method, in the vocabulary of [9]. The direct problem consists in finding the Lie symmetries of a given set of constitutive equations, as developed by several authors in the field of Mechanics of Materials, see e.g. [16,21]. It allows a priori the calculation of all symmetries and associated invariances of those equations, which may lead to geometrical transformation rules revealing (graphical) superpositions of the material responses (the superposition of those responses in a given space of variables gives the master curve). The methodology at the root of this construction has been summarized in algorithmic form into four main steps in the diagram of Fig. 1. In the first step of the algorithm, one looks for one or several Lie symmetries formulated from experimental master curves. Next, the subsequent symmetry conditions are applied to a general expression of the constitutive equations involving some unknown functions, resulting in a PDE system satisfied by these unknown functions. The formulation of the constitutive equations (step 3) 
may follow two alternative routes, one relying on the calculated invariants associated with a given symmetry generator (in this case, only one generator is applied to one or several constitutive equations), and the other one based on the direct solution of the symmetry equations. The comparison of the constructed constitutive model with experimental results allows to validate or refine the model if needed. As a fourth and last step, the inverse problem is linked to the direct problem: once a possible material constitutive law has been constructed, the material's response can be predicted outside the range of variation of the control variables involved in the measurements for varying experimental conditions, hence using Lie symmetries inherent to the obtained constitutive law as an extrapolation technique. Master curves associated with those evidenced invariants can be built in various planes of analysis resulting from the planar projection of the evidenced invariants (choosing a suitable pair of variables). In addition to this, charts of the material's response predicted in this way - the iso- $t_{R}$ curves have been calculated in the $(T, \sigma)$ plane in the present contribution - have a high practical importance, in that they determine the range of admissible variations of the considered set of variables. The present methodology exploiting Lie symmetries in a combined direct and inverse manner can potentially be applied to a wide class of materials including polymers, metals, ceramics, metallic and polymeric foams and constitutive laws, such as nonlinear elasticity, viscoelasticity and viscoplasticity, with or without damage.

As concerns the obtained constitutive equations, it is further interesting to confront the obtained creep constitutive model to the classical models of the literature. Let us recall that in [24], creep constitutive models are classified into four main families, a brief summary being given in Table 6 .

Table 6. Some creep laws encountered in the literature.

\begin{tabular}{ll} 
Relationship & Reference(s) \\
\hline$\varepsilon=f_{1}(\sigma) f_{2}(t) f_{3}(T)$ & {$[20,25]$} \\
$\dot{\varepsilon}=f_{1}(\sigma) f_{2}(t) f_{3}(T)$ & {$[4,7,17,20]$} \\
$\dot{\varepsilon}=g_{1}(\sigma) g_{2}\left(\varepsilon_{c}\right) g_{3}(T)$ & {$[7,13,20]$} \\
$E(t) \Phi[\varepsilon(t)]=\sigma(t)+\int_{0}^{t} K(t, \tau) \sigma(\tau) d \tau$ & {$[20]$}
\end{tabular}

The first family of models is the total strain model, originally developed in [25] and in [20]. There the authors assume at constant temperature a straightforward relationship between the strain, stress and time (see Table 6). This amounts assuming that the isochronous creep curve at the time $t$ may be obtained by the multiplication of the instantaneous stress-strain curves by a function of time, viz $\sigma=\sigma_{i}(\varepsilon) \Psi(t)$. Another family of creep model is the time hardening model proposed in [7] and 
developed in $[4,17,20]$, for which one assumes that at constant temperature, there exists a relationship between the creep strain rate, the stress and the time.

Similarly, the strain hardening model considers a relationship between the creep strain rate, the stress and the cumulated creep strain $p$ at constant temperature, with $p=\varepsilon-\varepsilon_{e}=\varepsilon_{p}+\varepsilon_{c}$ (the variables $\varepsilon_{e}, \varepsilon_{p}$ and $\varepsilon_{c}$ denote the elastic, plastic and creep strain respectively). This concept was suggested in [7,13], and used by [20] who developed the hypothesis of the state equation. According to [20], the analytic form of these equations which allows the description of the first two parts of the creep curve writes

$$
\dot{p} p^{\alpha}=f(\sigma) \exp \left(-\frac{\Delta H}{R T}\right)
$$

Various useful suggestions for the stress dependence may be introduced, as the famous relation $f(\sigma)=k \sigma^{n}$, see [12]. The fourth class of model is the Rabotnov's nonlinear hereditary model meaning that the entire history of the stress prior to time $t$ affects the strain response at time $t$ (see Table 6). The kernel function $K(t, \tau)$ (or $K(t-\tau)$ ) characterizes the material properties, while the term $\Phi[\varepsilon(t)]$ stands for the nonlinear function of strain which is obtained from the uniaxial tensile test.

Complementary to the previous approaches, the authors of [23] have tested the validity of four constitutive equations for the description of creep of several metallic alloys (1.25Cr-0.5Mo-Si, 2.25Cr-1Mo and SUS316 steels), namely the power law, the exponential, logarithmic and Blackburn models, expressed respectively as

$$
\begin{aligned}
& \varepsilon=\varepsilon_{i}+a t^{b}+\dot{\varepsilon}_{M} t \\
& \varepsilon=\varepsilon_{i}+a[1-\exp (-b t)]+\dot{\varepsilon}_{M} t \\
& \varepsilon=\varepsilon_{i}+a \log [1+b t]+\dot{\varepsilon}_{M} t \\
& \varepsilon=\varepsilon_{i}+a[1-\exp (-b t)]+c[1-\exp (-d t)]+\dot{\varepsilon}_{M} t .
\end{aligned}
$$

The quantities $\varepsilon, \varepsilon_{i}, t, \dot{\varepsilon}_{M}$ therein represent successively the strain, the initial strain, the creep time and minimum creep rate; $a, b, c$ et $d$ are constants. The authors show that none of those model is able to account for the creep relation on the long durations encountered during service life, although the power law gives a better agreement with measurements. In the same group of researchers, the authors of [11] confirmed in a recent work that the power law is more adequate for $9 \mathrm{Cr} 1 \mathrm{Mo}$. If we consider the mathematical sructure of equation (58), one can notice that the creep strain rate is a power function of the stress, the time, and the strain. Consequently, our model can be viewed as derived from the second class of constitutive equations presented in [24], showing the coherence with those previous models. 
Despite of the good conformity of the present model with the previous laws, a discussion on the proposed systematic methodology and its consequences is in order. At first, let us notice that the formulation of "observed" Lie symmetries relies on the assumption that the transformation equations (11) are valid for a continuous variation of the parameter $\mu$ in $\left[\mu_{\min } ; \mu_{\max }\right]$, although only a couple of experimental values have been reached in this interval. This interpolation assumption seems to be reasonable but its extension to situations for which $\mu$ is not in the interval $\left[\mu_{\text {min }} ; \mu_{\text {max }}\right]$ (extrapolation) can be debatable. In the present model, the two master curves have been validated for $T$ between $748 \mathrm{~K}$ and $873 \mathrm{~K}$ and for $\varepsilon$ between $0.1 \%$ and $5.0 \%$. If the temperature $T$ is higher than $873 \mathrm{~K}$, or if values of strain greater than $5.0 \%$ are considered, new internal phenomena may be activated (e.g. damage, tertiary creep), and the validity of the transformations rules revealed by equations (36) and (41) can be discussed. More generally, one cannot guarantee a priori that microstructure effects activated in some range of variables $(T, \varepsilon, \sigma$, ...) are the same outside this range, or that other mechanisms may operate. In the same spirit, let us recall that contrary to the so-called time-temperature equivalence principle - which can be interpreted in terms of free volume - no physical modelling of the shift factors has been proposed. The transformation rules of equations (36) and (41) and the associated "observed" symmetries are presently viewed as mathematical tools to formulate constitutive equations.

Another limitation of the proposed algorithm is related to the non-uniqueness of master curves, which has to be examined at two levels of modelling. First, one should keep in mind that the adequate choice of variables (classically $\log \sigma$ vs $\log t$, or $\log \varepsilon$ vs $1 / T$ ) to be plotted from experimental data (step 1 of Fig. 1) is not obvious a priori, since master curves can only emerge for well-chosen representation variables.

The second item concerns the case of a single representation plane, for which the construction of a unique master curve can be problematic. For instance, let us consider in the plane $(x, y)$ the two lines $L_{1}$ defined by $y=\omega x+\beta_{1}$ and $L_{2}$ defined by $y=\omega x+\beta_{2}$. Since $L_{1}$ and $L_{2}$ have the same slope, it is either possible to superpose $L_{2}$ on $L_{1}$ horizontally, i.e. by translating $L_{2}$ along $x$ by $\left(\beta_{1}-\beta_{2}\right) / \omega$, or vertically by shifting $L_{2}$ along $y$ by the quantity $\beta_{1}-\beta_{2}$.

The choice of transformation rules (11) may have a great impact on the mathematical formulation of the constitutive equations, but no general "theorem" for choosing between several master curves can be formulated. In the same spirit, let us also notice that only classical Lie groups have been used in the present algorithm (translation and dilatation groups). The use of more sophisticated groups (e.g. rotation) is currently being studied. 


\section{References}

[1] Anderson P., Bellgardt T. and Jones F., Creep Deformation in a Modified 9Cr-1Mo Steel, Materials Science and Technology 19 (2003) 207-213.

[2] Barenblatt G., Scaling, Self-Similarity and Intermediate Asymptotics, Cambridge University Press, Cambridge 1996.

[3] Barenblatt G., Scaling Phenomena in Fatigue and Fracture, International Journal of Fracture 138 (2006) 19-35.

[4] Boyle J. and Spence J., Stress Analysis of Creep, Butterworth Publishers, Stoneham 1983.

[5] Carpinteri A. and Paggi M., A Unified Interpretation of the Power Laws in Fatigue and the Analytical Correlations Between Cyclic Properties of Engineering Materials, International Journal of Fatigue 31 (2009) 1524-1531.

[6] Chevali V., Dean D. and Janowski G., Flexural Creep Behavior of Discontinuous Thermoplastic Composites Non-Linear Viscoelastic Modeling and Time-Temperature-Stress Superposition, Composites: Part A 40 (2009) 870877.

[7] Davenport C., Correlation of Creep and Relaxation Properties of Copper, Appl. Mech. 5 (1938) 53-60.

[8] Dobes M. and Milicka K., The Relation Between Minimum Creep Rate and Time to Fracture, Metal Science 10 (1976) 382-384.

[9] Ganghoffer J.-F., Magnenet V. and Rahouadj R., Relevance of Symmetry Methods in Mechanics of Materials, Int. J. Eng. Math. 66 (2010) 103-119.

[10] Kim G., Kim S. and Ryu W., Evaluation of Monkman-Grant Parameters for Type 316LN and Modified 9Cr-Mo Stainless Steels, KSME Int. J. 16 (2002) 1420-1427.

[11] Kimura K., Kushima H. and Sawada K., Long-Term Creep Deformation Property of Modified 9Cr-1Mo Steel, Materials Science and Engineering A 510-511 (2009) 58-63.

[12] Lemaitre J. and Chaboche J.-L., Mécanique des Matériaux Solides, 2nd Edition, Dunod, Paris 2004.

[13] Ludwik P., Elemente der Technologischen Mechanik, Springer, Berlin 1909.

[14] Magnenet V., Rahouadj R. and Ganghoffer J.-F., A New Methodology for Determining the Mechanical Behavior of Polymers Exploiting Lie Symmetries: Application to a Stick-Like Material, Mechanics of Materials 41 (2009) 10171024.

[15] Olver P., Application of Lie Groups to Differential Equations, Springer, New York 1989. 
[16] Ozer T., Symmetry Group Analysis and Similarity Solutions of Variant Nonlinear Long-Wave Equations, Chaos, Solitons and Fractals 38 (2008) 722730.

[17] Penny R. and Marriott D., Design for Creep, McGraw-Hill, New York 1971.

[18] Phaniraj C., Choudhary B., Bhanu Sankara Rao K. and Raj B., Relationship Between Time to Reach Monkman-Grant Ductility and Rupture Life, Scripta Materialia 48 (2003) 1313-1318.

[19] Povolo F., Comments on the Monkman-Grant and the Modified MonkmanGrant Relationships, J. Materials Science 20 (1985) 2005-2010.

[20] Rabotnov Y., Creep Problems in Structural Members, North-Holland, Amsterdam 1969.

[21] Sahin D., Antar N. and Ozer T., Lie Group Analysis of Gravity Currents, Nonlinear Analysis: Real World Applications 11 (2010) 978-994.

[22] Saint-Antonin F., Essais de Fluage, Techniques de l’Ingénieur M140 (1995) $1-30$.

[23] Sawada K., Tabuchi M. and Kimura K., Analysis of Long-Term Creep Curves by Constitutive Equations, Materials Science and Engineering A 510-511 (2009) 190-194.

[24] Skrzypek J. and Hetnarski R., Plasticity and Creep, CRC Press, Boca Raton 1993.

[25] Soderberg C., The Interpretation of Creep Tests for Machine Design, Trans. ASME 58 (1936) 733-743.

[26] Totemeier T., Tian H. and Simpson J., Effect of Normalization Temperature on the Creep Strength of Modified 9Cr-1Mo Steel, Metallurgical and Materials Transactions A 37 (2006) 1519-1525.

[27] Viswanathan R., Damage Mechanisms and Life Assessment of HighTemperature Components, ASM International, USA, 1989.

Vincent Magnenet

LaEGO, EA 1145

Nancy-Université

Rue du Doyen Marcel Roubault, B.P. 40

54501 VANDOEUVRE-lès-NANCY

FRANCE

E-mail address: vmagnenetefree.fr 
Rachid Rahouadj

LEMTA, UMR-CNRS 7563

Nancy-Université

2 Avenue de la Forêt de Haye, B.P. 160

54504 VANDOEUVRE-lès-NANCY

FRANCE

E-mail address: rachid.rahouadjeensem.inpl-nancy.fr

Jean-François Ganghoffer

LEMTA, UMR-CNRS 7563

Nancy-Université

2 Avenue de la Forêt de Haye, B.P. 160

54504 VANDOEUVRE-lès-NANCY

FRANCE

E-mail address: jean-francois.ganghofferdensem.inpl-nancy.fr 